\title{
Single-file Movement of Ants Stressed by a High Temperature
}

\author{
Qiao Wang ${ }^{1,2}$, Weiguo Song1,*, Shujie Wang ${ }^{1}$, Siuming Lo ${ }^{2}$ \\ ${ }^{1}$ State Key Laboratory of Fire Science, USTC \\ No.96, JinZhai Road Baohe District, Hefei, China \\ qw2014@mail.ustc.edu.cn; wgsong@ustc.edu.cn; sj573@mail.ustc.edu.cn \\ ${ }^{2}$ Department of Architectural and Civil Engineering, CityU \\ Tat Chee Avenue, Kowloon, Hong Kong SAR, Hong Kong, China \\ bcsmli@cityu.edu.hk
}

\begin{abstract}
Single-file movement is a universal pattern in both nature and human society. In this paper, we investigate single-file movement of ants (Camponotus japonicus) driven by a high temperature in a narrow channel. Here, ants were placed in a chamber. The chamber was connected to a narrow channel which was $10 \mathrm{~cm}$ long and $0.6 \mathrm{~cm}$ wide so that the ants can escape through it one by one. Both chamber and narrow channel were in high temperature environment. In the channel, the random pause was observed due to the characteristic of ants. Moreover, ants were inclined to following the preceding one and trying to overtake it, which is different from the movement in natural investigation. On the other hand, the speed increased with distance headway when the distance headway is less than $0.26 \mathrm{~cm}$, that is less than the body size of an ant. Furthermore, touching phenomenon was observed. When the following ants touched the preceding one, they could reduce speed, stop or move backward. On the contrary, the preceding ants increased their speed. Thus, the touching effect in multiple ants experiment can enhance the evacuation efficiency.
\end{abstract}

Keywords: single-file movement, ant, high temperature, speed-distance headway, touching effect

\section{Introduction}

Single-file movement, which is one of the simplest system for exploring the relationship between velocity and density, had been studied by conducting controlled experiments with pedestrians [1]. In the experiments of single-file pedestrian movement [2], it was found that velocity had a linear relation with the inverse of density. For the different agents such as pedestrians, bicycles and cars, a consistent flow-density relation could be obtained after simple rescaling [3]. In the field of vehicle traffic, single-file is replaced by single-lane, and models [4-7] and experiments [8,9] have been conducted to study single-lane movement in vehicle traffic.

Similar with pedestrian and vehicle traffic, the single-file movement in natural traffic occurs frequently, for example in case of ants. Ants, which are social insects, have a trail system that is similar to vehicle traffic. This has drawn many researchers' attention [10]. In the process of foraging, ants move forward along the pheromone that is dropped on the ground by the leaders [11] so that the single-file movement is formed. Through observing ants' behaviors carefully, researchers indicated that a clear lane segregation formed (segregated into inbound and outbound) during the foraging process, in order to avoid head-on collisions [12]. Thus, unidirectional flow is formed in ant traffic. In collective unidirectional movement [13], average velocity did not rely on density and its flow increases monotonically with the density. Ants, with their small size and relatively less aggressiveness, are easy to be controlled. Therefore, they are available for controlled experiments.

In an controlled experiment, faster-is-slower effect and mass behavior are common phenomena of escape panic [14], which is different from the behavior of crowd under normal conditions. Thus it is interesting to study the behavior of ants under emergency conditions. Considering the influence of stress produced by citronella, Soria et al. [15] performed experiments of the carpenter ant Camponotus mus (Roger) to study the "Faster-is-Slower (FIS)" effect. In their work, the evacuation of ants was compatible with FIS effect because of a minimum evacuation time observing at intermediate citronella concentration. 
However, FIS effect of ants was different from the result presented in social force model [16]. In the process of escaping from a chamber with stimulation of citronella, no jamming and clogging appeared near an exit and the exponential decay of the frequency distribution of time intervals could be observed [17]. In addition, high temperature, as a new aversive stimuli for ants, was used to stress ants escaping [18]. In this work, authors observed that higher temperature resulted in faster evacuation.

Many researchers studied single-file movement of pedestrians, bicycles and cars through experiment and observation $[1-3,9,19]$. Nevertheless, the single-file movement of ants in stressed conditions was studied rarely. In the experiment conducted by Wang et al. [20], the speed of ants driven by citronella was approximately constant with the increasing density in a straight passageway.

In this work, we will further investigate the single-file movement of ants under stressed conditions and compare the difference between individual and collective motion. Here, high temperature is used to drive ants to pass through a narrow channel. Both single-file movement of multiple ants and single ant movement are investigated.

\section{Materials and Methods}

\subsection{Ants}

A colony of Camponotus japonicus was captured in the wild in Shaanxi Province, China, and then transported to our laboratory using centrifuge tubes. This colony with approximately 500 workers was placed in a non-transparent plastic box $(51 \mathrm{~cm}$ long, $35 \mathrm{~cm}$ wide and $33 \mathrm{~cm}$ high) that was coated by Fluon to prevent ants from escaping. The box was shaded by a lid on it. Ants were raised in a box with a constant temperature $\left(24 \pm 2{ }^{\circ} \mathrm{C}\right)$ and suitable humidity under natural light/dark cycles. When no experiment is conducted, ants can move freely in the box with sufficient food (honey) and water supplying.

The length of Camponotus japonicus is usually between 0.8 and $1.4 \mathrm{~cm}$. In this experiment, we only adopted the workers whose body size was more than $1 \mathrm{~cm}$.

\subsection{Experimental Device}

We built a chamber connected to a narrow channel which was $10 \mathrm{~cm}$ long and $0.6 \mathrm{~cm}$ wide on the testbed, as shown in Fig. 1. The chamber resembles a funnel that is contributed to guide ants into channel. The $10 \mathrm{~cm}$ long channel ensures that the temperature in whole channel can stress ants to left this area. Considering the size of ants' limbs and antennae, the width of the channel was set to be 0.6 $\mathrm{cm}$ to avoid the phenomena of crawling side by side and U-turn motion. The chamber and channel composed of tasteless rubber were $0.6 \mathrm{~cm}$ high. They were covered by a PMMA (polymethyl methacrylate) lid, in order to prevent ants overlapping and record the experiment by video mounted over the device. A hole was existed in the lid to guide ants into the chamber. The bottom of the device was a copper plate, and there were two copper pipes under two ends of the plate. Water heated by two thermostatic bathes to different temperatures was poured into these two pipes to heat the plate. In this way, a temperature gradient formed as shown in Fig.1 (b), which could successfully induce the ants to escape from the chamber and move in channel one by one spontaneously. Hence, the entrance of the narrow channel was at a high temperature, the end of the channel (i.e., the final exit of the chamber) was at a low temperature. As the temperature of the chamber was high, it could hurt ants for a long time stay. Thus, this high temperature could drive ants to enter the unidirectional channel and form single-file movement. 


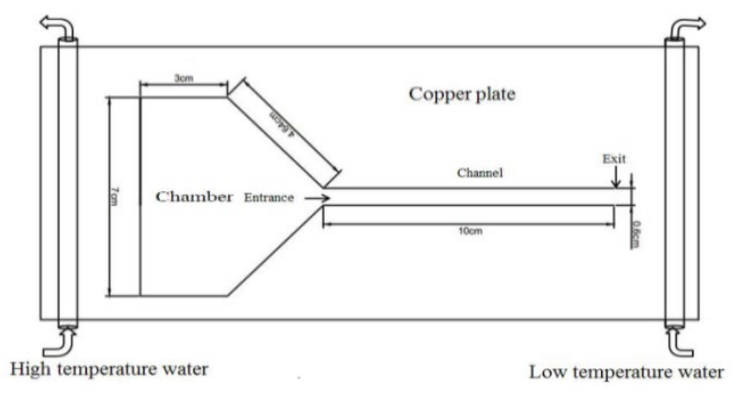

(a)

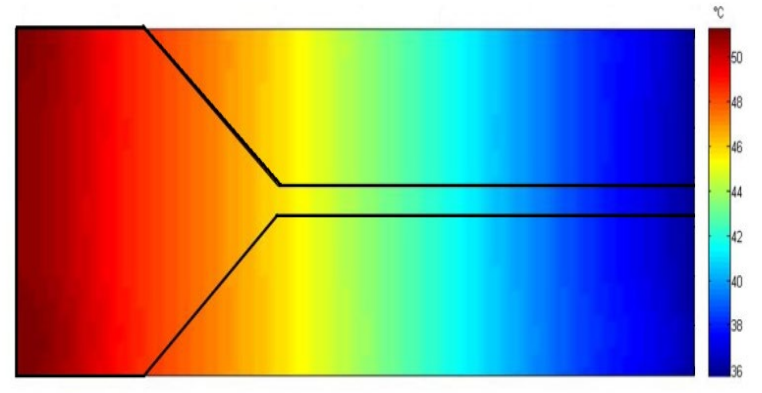

(b)

Fig. 1. Experimental device. (a) the investigation region is the unidirectional channel which is $10 \mathrm{~cm}$ long and 0.6 $\mathrm{cm}$ wide, and the chamber resembles a funnel with an area of $33.5 \mathrm{~cm}^{2}$. (b) temperature gradient of chamber and narrow channel is shown.

\subsection{Experimental Protocol}

The experiment comprised of two parts: single-file movement of multiple ants and single ant movement. Each ant was exposed to a high temperature only once. First, the room temperature was $23{ }^{\circ} \mathrm{C}$, and the entrance of the narrow channel was blocked. Then, two thermostatic bathes were employed to heat the water to different temperatures. Highest and lowest temperatures of water were respectively 75 ${ }^{\circ} \mathrm{C}$ and $30{ }^{\circ} \mathrm{C}$. After two temperatures of the water were satisfied, it was used to heat the copper plate circularly. Consequently, highest temperature in the chamber was $51^{\circ} \mathrm{C}$, and temperatures at the entrance and exit of the channel were $45.8{ }^{\circ} \mathrm{C}$ and $35.8{ }^{\circ} \mathrm{C}$, respectively. Therefore, temperature gradient was formed in the chamber and channel. When the temperature gradient of chamber and channel was stable, 15-30 ants with a similar size were quickly guided into the chamber, and then a transparent plastic sheet was used to cover the hole. Finally, we opened the entrance of the narrow channel, and began to record single-file movement of ants. The moment of opening the entrance was the beginning of the experiment. In the experiment of single-file movement of multiple ants, when all normal ants (besides injured ants) passed the exit of the channel, one trial was finished. This experiment was repeated 14 times under the same conditions. The movement of ants in the narrow channel was recorded by a digital video camera (digital sampling rate is $25 \mathrm{fps}$ ). Image analysis was implemented through the slow-motion mode of video-playing software. The coordinates of ants' heads in the narrow channel were extracted every two frames (0.08 seconds).

In the experiment of single ant movement, only one ant was introduced into the chamber in each trial. A total of 18 tests were performed. Each trial started when the ant entered the chamber, and was accomplished when the ant left the channel or was injured in the chamber.

\section{Results and Discussion}

\subsection{Single-file Movement of Multiple Ants}

In the light of the size measurements of 88 selected ants randomly, the body size is $1.23 \pm 0.14 \mathrm{~cm}$ (average \pm standard deviation). The temporal evolution of each ant passing through the narrow channel was extracted from video records and in this way the coordinate of a head of an ant was obtained. Due to the restriction of width of channel, ants moved in the narrow channel with one-dimensional motion. Thus, we calculated the instantaneous speed in $\mathrm{x}$-axis every eight frames $(0.32$ seconds $)$ to relieve data fluctuation and data error caused by too short time interval. The instantaneous speed $V(t)$ at time $t$ is given by

$V(t)=\frac{S(t+\Delta t / 2)-S(t-\Delta t / 2)}{\Delta t}$

where $S(t)$ denotes the abscissa of an ant's head, namely the moving distance in $t$ seconds in the narrow channel. $\Delta t$ represents the time interval which equals to 0.32 seconds in equation (1). In order to exclude outliers, pauta criterion was used in speed statistics for all ants. 
In the field of vehicle traffic [9] and pedestrian [21] dynamics, the speed increases with distance headway until the desired speed is reached. In this work, we are also interested in the relationship between speed and distance headway that is defined as the distance from one ant's head to the tail of a preceding ant. When ants entered the channel, they moved in the channel one by one due the restraint of the width of channel. The instantaneous speed for the leader ant, preceding ants and following ants and the distance headway between two successive ants evolution with time are plotted in Fig. 2. The scattered dots represent the instantaneous speed for each time. Then these dots were connected by a smooth curve with Savizky-Golay method, from which the variation trend of speed with time can be obtained. For the leader ants, there is no interference in front, and the instantaneous speed is influenced by some unknown reasons that cannot be observed from video, which resulted in random pause or deceleration. As shown in Fig. 2(a), the instantaneous speed of leader ants is varying with time. Moreover, the value of the speed has larger fluctuation. Similar with leader ants, the instantaneous speed of following ants changes with time. When the distance headway is large enough, the following ants reduce their speed sometimes. On the other hand, the speed of following ants is influenced by not only some unknown reasons, but also the distance headway as illustrating in Fig. 2(b). From Fig. 2(b), we can see that the following ant decreased the speed once the distance headway is approximate to zero. However, in other case, if the distance headway is less than zero, which means the head of following ant overtakes the tail of preceding one, the following ants could also continue to move ahead as displayed in Fig. 2(c).

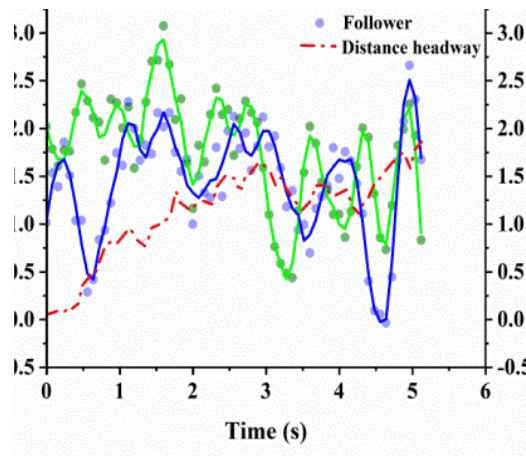

(a)

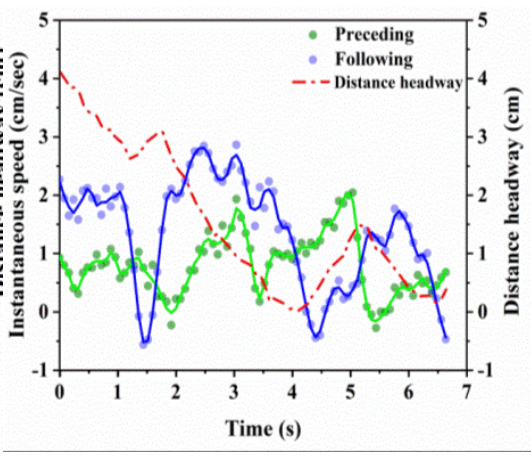

(b)

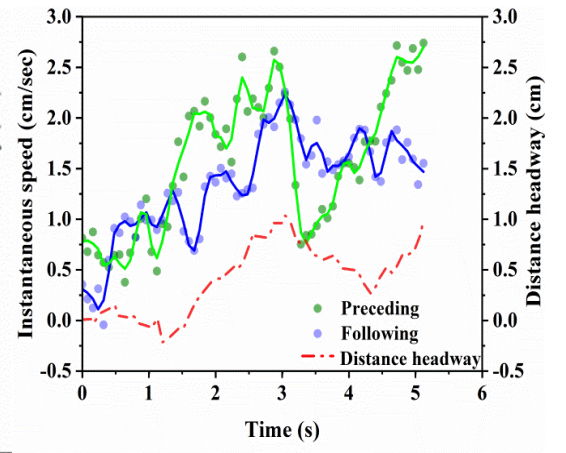

(c)

Fig. 2. The time-series of instantaneous speed (green dots for preceding ants and blue dots for following ants) and the distance headway (red dashed line). (a) shows the first ant in one trail and it's follower. (b) and (c) display two successive ants in middle of experiment process.

The results obtained from Fig. 2 indicate that the value of distance headway is less than zero for escaping quickly in some cases. Here, we counted the frequency distribution of distance headway for all the ants moving in the channel as shown in Fig.3. By using the Gaussian fit, the average distance headway $\mu$ is $0.3 \mathrm{~cm}$, and the standard deviation $\sigma=0.32$. This result implies most ants prefer to follow their preceding one closely when passing through the channel, as they are urgent to escape from the high temperature area. In addition, negative values of distance headway also account for a certain proportion. 


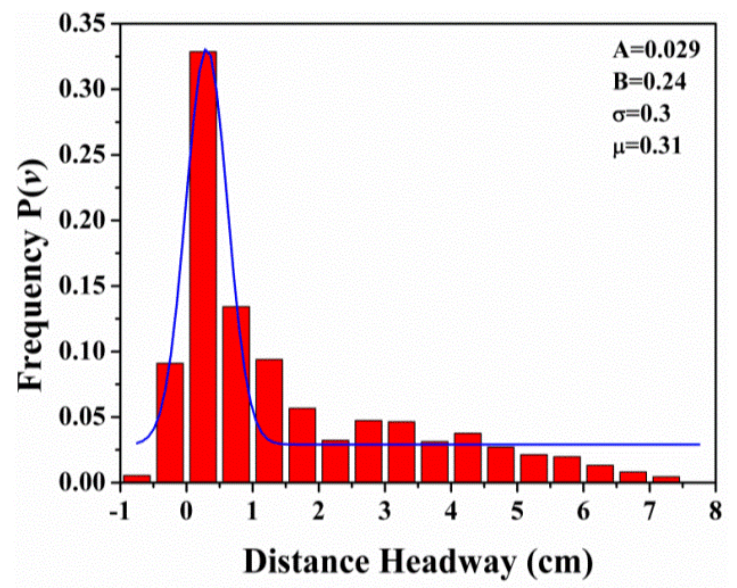

Fig. 3. Distance headway frequency distribution of all ants. The blue solid line represents the Gaussian fitting The coefficient of determine $\mathrm{R}^{2}$ is 0.894 .

Due to the stress caused by the high temperature, the following ants tried to overtake the preceding one. Thus, following ants were apt to follow the leader one closely, namely less distance headway existed between them. As shown in Fig. 4, there are some special discrete points with distance headway $<0$ or speed $<0$. During the experimental process, it was observed that some ants (which we call activists) preferred to overtake its front one when the front one moved with low speed or stopped, in order to escape from the chamber quickly. However, they failed because of the limitation of the channel width. In this case, they can move with the uniform speed sequentially by following the preceding one, as a result of their heads paralleled with the tail of preceding ant. On the other hand, some other ants named as pacifists just decelerated or moved backward once they touched the preceding one. Hence it can be explained that when the distance headway is less than 0 , the speeds have both negative and positive values. In addition, we also observed the backward motion (speed $<0$ ) in spite of large available distance which existed in front. Under stressed conditions, it is common for the ants that the distance headway is not more than zero.

The instantaneous speed of a single ant displayed in Fig. 4 is scattered whatever the distance headways are, which is consistent with the result shown in $[13,20]$. In order to obtain the relation of the speed-distance headway, we applied a binning procedure [22] to this scattered data, i.e. dividing the whole data of speed into a series of bin per $0.1 \mathrm{~cm} / \mathrm{sec}$, and then calculating the peak value of frequency histogram that is obtained from the data of the distance headway falling into each bin firstly. Next, we fitted the peak values by means of linear fitting. The results presented in Fig. 4 show that the speed increases with the distance headway when the value of distance headway is less than $0.26 \mathrm{~cm}$. The result of the linear fitting is 6.4 for the slope. The inverse of slope that is interpreted as a sensitivity to the distance headway [22] is 0.156 in present work. It indicates that ants are sensitive to distance headway. When the distance headway is small, ants can move with both high speed and low speed, and they have strong ability to control their speed. 


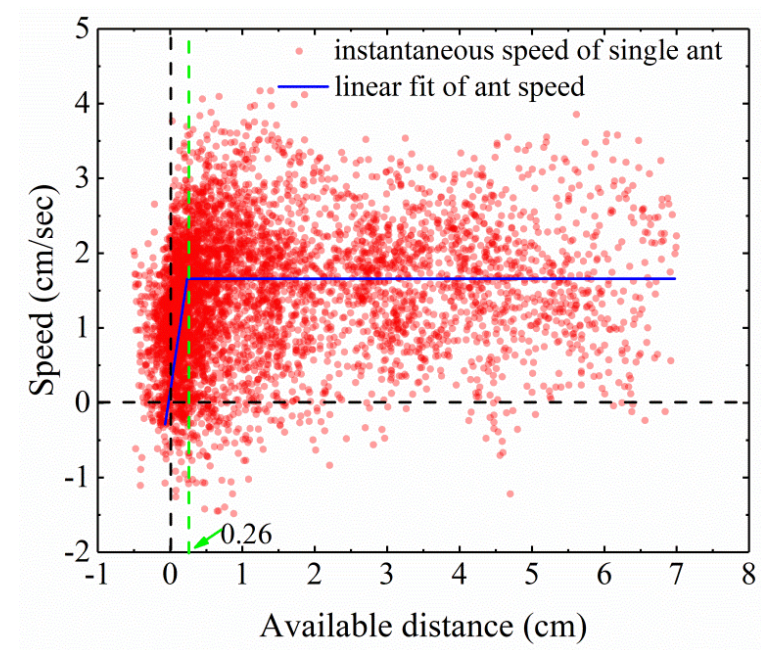

Fig. 4. Relationship between speed and distance headway. The fitting results are $y=6.4^{*} x$ and $y=1.65 . y$ and $x$ represent speed and distance headway respectively.

\subsection{Comparison of Single Ant and Single-file Movement Experiment}

In single-file movement experiment, multiple ants were used to investigate the collective motion in the narrow channel. We also employed single ant experiment to study individual motion with the same condition. The single ant movement using the same protocol with single-file movement experiments was conducted. It was repeated 18 times and 15 ants succeed in escaping from the chamber through the narrow channel. Three ants were injured because of long stay in high temperature, which resulted in failed escape. In order to investigate the single ant movement and compare the results with single-file movement of multiple ants, speeds of single ant were obtained with the same methods as above.

Single ant entered the channel driven by high temperature, and in single ant experiment, for each trial, only one ant moved in channel. The relations of speed-time for three ants are plotted in Fig. 5. From this figure it can be seen that the instantaneous speed of ant is fluctuant with time. Sometimes, ants moved in channel with high speed, but after a while, they decreased their speed to zero, even moved backward. The specific reason needs to be studied furtherly in the future.

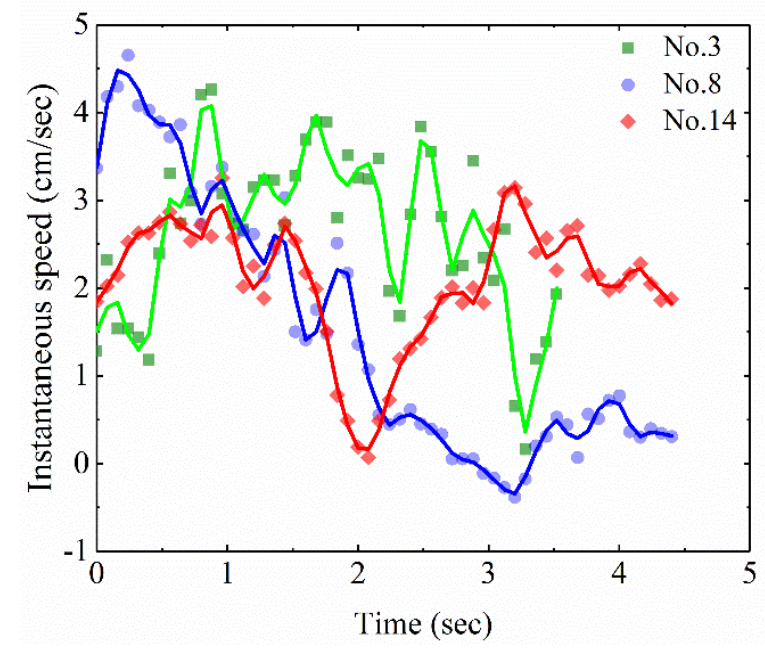

Fig. 5. Variation of speed with time for three single ant in the channel.

First of all, we compared the average speed for both single-file movement and single ant experiments. The speed distributions of all ants moving in the narrow channel for single ant and single-file movement experiments satisfy the Gaussian distribution, as illustrated in Fig. 6. The average speeds of these two experiments are 1.48 and $0.85 \mathrm{~cm} / \mathrm{sec}$, respectively. This comparison of the results manifests that the 
multiple ants moved in channel can improve the average speed of ants. In the process of experiment, touching phenomenon was observed in single-file movement experiment in which the ants were stressed by high temperature. Attempting to escape from the chamber quickly, it is observed that the following ant touched (expressing as pushing behavior) the preceding one and tried to overtake it if the preceding ant was slower. This pushing behavior led to the increasing of speed of preceding ant. However, this phenomenon is not observed in natural trail of monomorphic ant [13]. From another point of view, the existence of touching behavior in single-file movement experiment is the reason that results in higher average speed $(1.48 \mathrm{~cm} / \mathrm{sec})$ than in single ant experiment $(0.85 \mathrm{~cm} / \mathrm{sec})$ in narrow channel. Generally, touching behavior improves the movement efficiency of passing channel.

The standard deviations of single ant and single-file movement experiments are 1.01 and 0.9 , which is obtained by means of Gaussian fitting. Actually, the standard deviation reflects the degree of dispersion among individuals in one group. Comparatively speaking, the standard deviation $(\sigma=1.01)$ in single ant experiments is larger than single-file movement experiments $(\sigma=0.9)$, which means that the movement of single ant in channel are more arbitrary. Without the pushing from the following ant, ants moved according to their own characteristic.

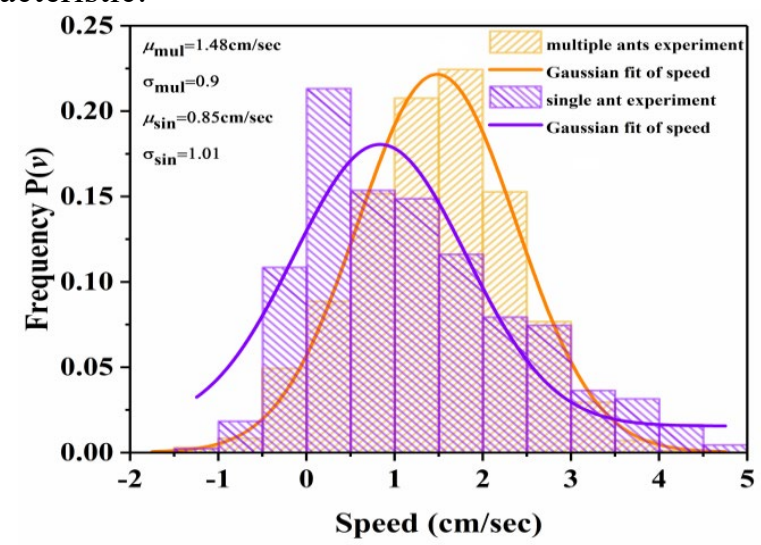

Fig. 6. Speed distribution of ants for single-file movement and single ant experiments.

Trajectories of ant moving in the channel both of single-file movement and single ant experiments are presented in Fig.7. All of the trajectories are tortuous, which signifies the moving ants do not walk forward along a straight line in the narrow channel. This finding is identical with the single-file movement of pedestrians [2]. However, the periodic oscillating was not observed from their trajectories. In these two experiments, the short pause and backward motion were observed in the trajectories of ants. On the other hand, in single ant experiment, there is significant difference between different ants for trajectories. As plotted in Fig. 7(b), the upper trajectory (blue line) displays a relatively smooth line, which presents a fluent movement process. Nevertheless, the bottom one (red line) shows a slow speed. From the bottom of Fig. 8(b), it can be seen that the ant hovered in the distance of 7 to $9 \mathrm{~cm}$ away from the entrance of channel for a long time.

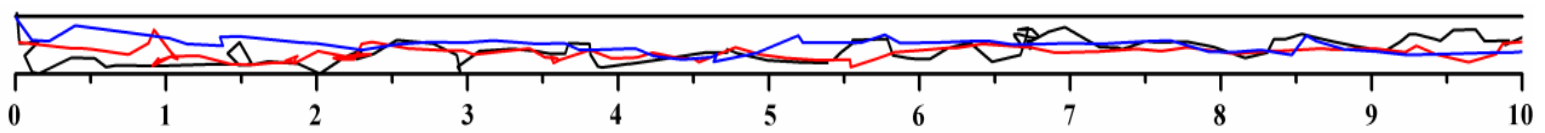

(a)

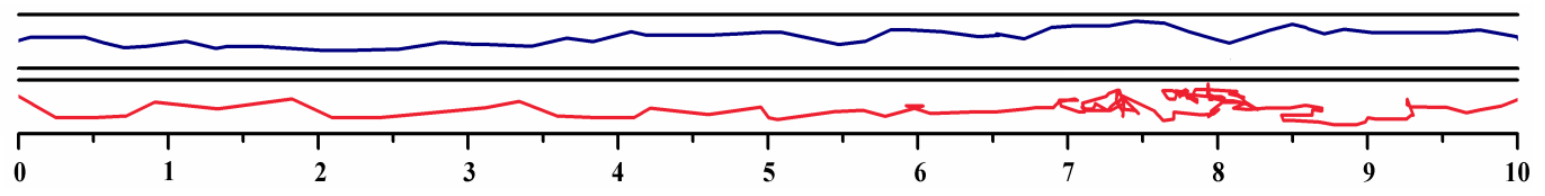

(b)

Fig. 7. Trajectories of ants in unidirectional channel for (a) single-file movement experiment and (b) single ant experiment. 
Summarily, through comparing these two experiments, it was found that the pushing behavior plays an important role on single-file movement experiment which can promote preceding ants to move ahead or increase their speed. By this way, the efficiency of evacuating from a chamber by passing a long channel has improved.

\section{Conclusions}

In this work, we investigated single-file movement and the interaction between ants in the narrow channel under high temperature conditions. Under the stimulation of high temperature, ants preferred to follow the front one closely for escaping quickly. In the channel, touching behavior played a main role on escape. It was expressed that the average speed of ants in the single-file movement experiment was faster than that in the single ant experiment. In addition, similar to pedestrian and traffic, the speed of ants was dependent on a certain critical distance headway. Here, the critical distance headway for ant is $0.26 \mathrm{~cm}$ due to high ability to control their speed. The trajectories of ants presented a wavy form, which is similar to pedestrian in single-file movement, and whether an amplitude of ants is decreasing with increasing speed like pedestrian needs to be studied in the future work.

Generally, the single-file movement of ants has common characteristics with pedestrians and traffic in some respects. In the future, it is necessary to make a quantitative comparison between ants and pedestrian and vehicle traffic in single-file movement. Furthermore, in the ant trail model studying the ant movement under dangerous conditions, the long body size of ant and touching effect observed in this experiment should be considered in the future.

\section{Acknowledgements}

This research was supported by Key Research and Development Program of China (2016YFC0802508), and Fundamental Research Funds for the Central Universities (WK2320000035).

\section{References}

[1] A. Seyfried, B. Steffen, W. Klingsch, and M. Boltes, "The fundamental diagram of pedestrian movement revisited," Journal of Statistical Mechanics: Theory and Experiment, vol. 2005, p. P10002, 2005.

[2] X. Liu, W. Song, and J. Zhang, "Extraction and quantitative analysis of microscopic evacuation characteristics based on digital image processing," Physica A: Statistical Mechanics and its Applications, vol. 388, pp. 2717-2726, 2009.

[3] J. Zhang, W. Mehner, S. Holl, M. Boltes, E. Andresen, A. Schadschneider, et al., "Universal flow-density relation of single-file bicycle, pedestrian and car motion," Physics Letters A, vol. 378, pp. 3274-3277, 2014.

[4] S. Wolfram, Theory and applications of cellular automata vol. 1: World scientific Singapore, 1986.

[5] A. Schadschneider, "The nagel-schreckenberg model revisited," The European Physical Journal B-Condensed Matter and Complex Systems, vol. 10, pp. 573-582, 1999.

[6] M. Fukui and Y. Ishibashi, "Traffic flow in 1D cellular automaton model including cars moving with high speed," Journal of the Physical Society of Japan, vol. 65, pp. 1868-1870, 1996.

[7] M. Bando, K. Hasebe, A. Nakayama, A. Shibata, and Y. Sugiyama, "Dynamical model of traffic congestion and numerical simulation," Physical review E, vol. 51, p. 1035, 1995.

[8] R. Herman and R. B. Potts, "Single lane traffic theory and experiment," 1900.

[9] G. F. Newell, "Nonlinear effects in the dynamics of car following," Operations research, vol. 9, pp. 209-229, 1961.

[10] E. Wilson, "The insect societies (Belknap, Cambridge, USA, 1971); B. Hölldobler and EO Wilson," in The ants, 1990.

[11] E. O. Wilson, "The insect societies. Belknap," Harvard, Cambridge MA, 1971. 
[12] I. D. Couzin and N. R. Franks, "Self-organized lane formation and optimized traffic flow in army ants," Proceedings of the Royal Society of London B: Biological Sciences, vol. 270, pp. 139-146, 2003.

[13] A. John, A. Schadschneider, D. Chowdhury, and K. Nishinari, "Trafficlike collective movement of ants on trails: Absence of a jammed phase," Physical review letters, vol. 102, p. 108001, 2009.

[14] D. Helbing, I. J. Farkas, and T. Vicsek, "Simulating dynamical features of escape panic," Nature, vol. 407, pp. 487-490, 2000.

[15] S. Soria, R. Josens, and D. Parisi, "Experimental evidence of the "Faster is Slower" effect in the evacuation of ants," Safety science, vol. 50, pp. 1584-1588, 2012.

[16] D. R. Parisi, S. A. Soria, and R. Josens, "Faster-is-slower effect in escaping ants revisited: Ants do not behave like humans," Safety science, vol. 72, pp. 274-282, 2015.

[17] S. Wang, W. Lv, and W. Song, "Behavior of Ants Escaping from a Single-Exit Room," Plos One, vol. 10, p. e0131784, 2015.

[18] S. Boari, R. Josens, and D. R. Parisi, "Efficient egress of escaping ants stressed with temperature," Plos One, vol. 8, p. e81082, 2013.

[19] U. Chattaraj, A. Seyfried, P. Chakroborty, and M. K. Biswal, "Modelling single file pedestrian motion across cultures," Procedia-Social and Behavioral Sciences, vol. 104, pp. 698-707, 2013.

[20] S. Wang and W. Song, "Experimental Study of Ant Movement in a Straight Passageway under Stress Conditions," Journal of Insect Behavior, vol. 29, pp. 735-743, 2016.

[21] W. Lv, Z. Fang, X. Wei, W. Song, and X. Liu, "Experiment and Modelling for Pedestrian Following Behavior Using Velocity-headway Relation," Procedia Engineering, vol. 62, pp. 525-531, 2013.

[22] A. Jelić, C. Appert-Rolland, S. Lemercier, and J. Pettré, "Properties of pedestrians walking in line: Fundamental diagrams," Physical review E, vol. 85, p. 036111, 2012. 